\title{
Cosmological Perturbations from a New-Physics Hypersurface
}

\author{
V. Bozza ${ }^{1,2,3}$, M. Giovannini ${ }^{4}$ and G. Veneziano ${ }^{4}$ \\ (1) Dipartimento di Fisica "E. R. Caianiello", Università di Salerno, \\ Via S. Allende, 84081 Baronissi (SA), Italy \\ (2) INFN, Sezione di Napoli, Gruppo Collegato di Salerno, Salerno, Italy \\ (3) Enrico Fermi Center, via Panisperna 89/A, 00184 Roma, Italy \\ (4) Theoretical Physics Division, CERN, CH-1211 Geneva 23, Switzerland
}

\begin{abstract}
Within a broad class of inflationary models we critically analyse the way initial quantum fluctuations on a new-physics hypersurface (NPH) affect standard predictions for large-scale cosmological perturbations. We find that these so-called transplanckian effects crucially depend on the definition of the "vacuum state" in particular on which Hamiltonian is minimized on the NPH in order to select such a state. Transplanckian effects can be made much smaller than previously suggested if sufficiently "adiabatic" Hamiltonians are minimized.
\end{abstract}


There is general consensus that presently observed CMB anisotropies, as well as the large-scale structure of our visible Universe, originate from the parametric amplification of quantum-mechanical fluctuations occurring during inflation (for a review, see e.g. [1]). Present large-scale experiments thus offer a window on physics at the energy scales at which inflation took place. Since the experimental value of CMB temperature fluctuations bounds the Hubble parameter during inflation by $10^{-6}-10^{-5} M_{\mathrm{P}}$, this implies that present largescale observations are sensitive to physics at energy scales that can be, at most, as large as $10^{-3} M_{\mathrm{P}} \sim M_{\mathrm{GUT}}$. While this is certainly a very interesting energy scale for particle physics, it is definitely below the scale of quantum gravity or, presumably, the scale characterizing new physics in any realistic string theory.

There is instead much debate $[2,3,4,5,6]$ about the possibility that present largescale experiments may test physics at even shorter scales, down to the string/Planck-length scales or even below. A particularly important issue is that of finding out the order of these "transplanckian" corrections in the (supposedly small) parameter $H / \Lambda$, where $H$ is a typical Hubble parameter during inflation and $\Lambda$ is the scale of some unknown new physics. Although large effects can result from assuming that the new physics breaks some wellestablished low-energy principles, one would like to establish the existence and order of magnitude of the effects in a more model-independent way.

An interesting step in this direction goes as follows [7] (see also [8]). Any scale of physical interest today was very tiny if blue-shifted back till the beginning of inflation. In fact, unless the duration of inflation is just the minimal one for solving the standard cosmological problems, even the largest relevant cosmological scales today were very much sub-Planckian at the beginning of inflation. To reinforce this statement, let us remark that the difference between initial classical and quantum fluctuations is that the former, by definition, are at super-Planckian length scales, and are therefore much beyond our horizon -hence unobservable- today, while the latter must have also been present at sub-Planckian scales. This characterization of the two kinds of initial perturbation is crucial if one wants to argue that inflation washes out any initial (and arbitrary) classical inhomogeneity while replacing it by a spectrum of fluctuations that is fully normalized by the uncertainty principle of quantum mechanics.

The problem with the above picture is that it forces one to normalize quantum fluctuation in a regime (the transplanckian one) where, in general, physics is not known. Furthermore, if the standard formula is used, say for tensor metric perturbations,

$$
\delta_{h}(k)=k^{3 / 2} h(k) \sim \ell_{\mathrm{P}} k / a,
$$

we see that the fluctuations of the metric become $O(1)$ as soon as $\omega \equiv k / a=M_{\mathrm{P}}{ }^{1}$.

In order to avoid the above difficulty, while preserving the crucial distinction between

\footnotetext{
${ }^{1}$ Note that this is not the case for tensor perturbations in pre-big bang cosmology [9], since the relevant scale factor is the one in the Einstein frame and blows up, rather than vanishes, at $t \rightarrow-\infty$.
} 
classical and quantum fluctuations, it can be argued, instead [8], that, while classical fluctuations are given for all scales in excess of some cut-off length $l=\Lambda^{-1}$ on one and the same initial space-like hypersurface, quantum fluctuations are created at all times, and should only be looked at, for each physical scale $\omega$, after an "initial" ( $k$-dependent) time defined by $\omega(t)=k / a(t)=\Lambda$. In other words, in this new way of looking at initial conditions, both classical and quantum fluctuations are only considered above the scale $\Lambda^{-1}$, but, while the former are given on a conventional space-like hypersurface, the latter are given $[7,8]$ on the unusual "new-physics hypersurface" (NPH) defined by $\omega(t)=\Lambda$, hence in a way that mixes coordinates and momenta.

If this point of view is taken the question arises of the possible effects of the NPH on present observations. It has been argued, for instance, that in the de Sitter case corrections in the two-point function of the metric fluctuations are linear in the ratio $H / \Lambda[7]$, computable, and possibly observable [10] (see also $[11,12,13]$ for different perspectives). While some aspects of this suggestion may be theoretically justified according to our previous discussion, the mere existence of a cut-off does not allow, in our opinion, to compute accurately any specific correction to observables unless the nature of the physics above the cut-off is, even roughly, specified. Suppose, for instance, that, for some reason, we take the initial state of the fluctuations on the NPH to be the one obtained from the unitary evolution of the state minimizing the Hamiltonian at $t \rightarrow-\infty^{2}$. In this case the two-point function will not have any correction related to the "initial" time $t_{0}(k)$ lying on the NPH.

Assume instead, as in $[7,10]$, that we do not know anything of the physics above $\Lambda$ or, if we prefer, about what happened to the past of the NPH. Logic would suggest that, in this case, very little, if anything, can be said about the corrections to the standard predictions. The simple technical reason for this is that, in order to compute correlation functions at some late time, we have to know the initial state on the NPH and this is all but impossible without some knowledge of the physics above the cut-off.

In this Letter we shall explore the sensitivity of these corrections to the choice of the initial state on the NPH. In the absence of a specific knowledge of ultra-high energy physics, we shall demand that such initial state minimizes the Hamiltonian on the NPH. If this were a unique prescription, such an assumption would lead to definite predictions on the form and the magnitude of the corrections. Unfortunately, in time-dependent problems, there is no conserved Hamiltonian and the possibility of performing time-dependent field-redefinitions leads to different Hamiltonians connected among themselves by simple canonical transformations. While the (classical or quantum mechanical) evolution is indeed independent of the choice of the Hamiltonian, the minimization of one or the other on the NPH leads to different initial states and, consequently, to different corrections in the power spectrum. As we shall see, in the argument of $[7,10]$ there is an implicit assumption that "transplanckian"

\footnotetext{
${ }^{2}$ Such an assumption may not be as crazy as it looks, particularly when, as $t \rightarrow-\infty$, space-time becomes trivial. There are presumably no transplanckian effects due to just having a single particle/string carry momentum in excess of $M_{\mathrm{P}}$, as is well known for the vertex operators of string theory in flat space-time.
} 
effects lead to the minimization of a particular Hamiltonian, the one that turns out to give "large" corrections. After a general discussion of the quantum mechanical evolution of the system we shall look at the consequences of minimizing various Hamiltonians characterized by their "adiabaticity" i.e. by the way they go to the free Hamiltonian as $t \rightarrow-\infty$. Amusingly, we will find a strict correlation between the degree of "adiabaticity" and the size of the corrections, suggesting that the latter should be much smaller than claimed in $[7,10]$ and possibly even smaller than argued by other authors [14].

Consider, for simplicity, a spatially flat Friedmann-Robertson-Walker geometry whose line element can be written, in conformal time, as

$$
d s^{2}=a^{2}(\eta)\left[d \eta^{2}-d \vec{x}^{2}\right]
$$

where $a(\eta)$ is the scale factor whose rate of variation $a^{\prime} / a$ will be denoted by $\mathcal{H}$ (we shall use a prime to denote derivatives with respect to conformal time).

Consider now, in the background (2), some metric fluctuations, for instance the tensor perturbations of the geometry having the nice feature of being decoupled from the fluctuations of the matter sources. The time evolution of each of the two tensor polarization will be obtained by perturbing to second order the gravity action, taken to be, for simplicity, of the Einstein-Hilbert form. The dynamics of each tensor polarization $h$ will then be described by $[15]$

$$
S=\frac{1}{4 \ell_{\mathrm{P}}^{2}} \int d^{4} x a^{2} \eta^{\mu \nu} \partial_{\mu} h \partial_{\nu} h,
$$

where $\ell_{\mathrm{P}}=M_{\mathrm{P}}^{-1}=\sqrt{8 \pi G}$ and $\eta_{\mu \nu}$ is the Minkowski metric.

Let us assume, following $[7,8]$, that the effective description of the tensor modes of the geometry provided by Eq. (3) is only valid up to some typical scale $\Lambda$ at which the laws of some new/unknown physics replace Eq. (3). This assumption amounts to demanding a cut-off on the physical momenta of the gravitons, i.e.

$$
k / a(\eta) \leq \Lambda
$$

where $k$ is the comoving wavenumber. In any kind of inflationary background, Eq. (4) is saturated at some time $\eta_{0}(k)$ defining the NPH on which initial conditions for the fluctuations are given.

As discussed above, owing to the time dependence appearing in the action (3), different Hamiltonians can be used to describe the evolution of the system. Defining, for the sake of simplicity,

$$
\Psi=\frac{h}{\sqrt{2} \ell_{\mathrm{P}}},
$$

a possible selection of different Hamiltonians can be the following

$$
H^{(1)}(\eta)=\int d^{3} x \frac{1}{2}\left[\frac{\Pi^{2}}{a^{2}}+a^{2}\left(\partial_{i} \Psi\right)^{2}\right],
$$




$$
\begin{aligned}
& H^{(2)}(\eta)=\int d^{3} x \frac{1}{2}\left[\pi^{2}+2 \mathcal{H} \psi \pi+\left(\partial_{i} \psi\right)^{2}\right], \\
& H^{(3)}(\eta)=\int d^{3} x \frac{1}{2}\left[\tilde{\pi}^{2}+\left(\partial_{i} \psi\right)^{2}-\frac{a^{\prime \prime}}{a} \psi^{2}\right],
\end{aligned}
$$

where

$$
\Pi=a^{2} \Psi^{\prime}, \quad \psi=a \Psi, \quad \pi=\frac{\Pi}{a}, \quad \tilde{\pi}=\psi^{\prime},
$$

are the relations between the different canonical fields. It is not difficult to check that it is possible to go from one Hamiltonian to the other through a suitable canonical transformation. For instance, the transformation $H^{(2)}(\eta) \rightarrow H^{(3)}(\eta)$ is generated, in a standard way, by

$$
\mathcal{F}_{2 \rightarrow 3}(\psi, \tilde{\pi}, \eta)=\int d^{3} x\left(\psi \tilde{\pi}-\frac{\mathcal{H}}{2} \psi^{2}\right),
$$

a functional of the old fields and of the new momenta $\tilde{\pi}$. By differentiating the generating functional, we obtain the relation between the old momenta (i.e. $\pi$ ) and the new ones, as well as a change in the Hamiltonian

$$
\begin{aligned}
& \pi=\tilde{\pi}-\mathcal{H} \psi, \\
& H^{(2)}(\psi, \pi, \eta) \rightarrow H^{(3)}(\psi, \tilde{\pi}, \eta)=H^{(2)}(\psi, \pi, \eta)+\frac{\partial \mathcal{F}_{2 \rightarrow 3}}{\partial \eta} .
\end{aligned}
$$

Bearing in mind Eqs. (10) and (11), the right-hand side of Eq. (12) leads exactly to Eq. (8). With similar considerations, all the Hamiltonians (6)-(8) can be related to one another by suitable canonical transformations.

Fully equivalent classical evolutions should be expected by solving the appropriate Hamilton equations with the Hamiltonians (6)-(8). This statement is also true at the quantum level (see e.g. [16]) because the different classical actions corresponding to Eqs. (6)-(8) only differ by total derivatives. Hence, thinking for instance in terms of a functional integral approach, the transitions amplitudes differ, at most, by a field-dependent phase since the total derivatives appearing in the classical action, once inserted in the path integral, can be explicitly integrated.

As an example, consider the case of $H^{(2)}(\eta)$ whose associated Lagrangian density is

$$
\mathcal{L}^{(2)}(\vec{x}, \eta)=\frac{1}{2}\left[\psi^{\prime 2}-2 \mathcal{H} \psi \psi^{\prime}+\mathcal{H}^{2} \psi^{2}-\left(\partial_{i} \psi\right)^{2}\right] .
$$

Comparing Eq. (13) to the Lagrangian density associated with $\left.H^{(3)}\right)(\eta)$, we can notice that they differ by a total derivative

$$
\mathcal{L}^{(3)}(\vec{x}, \eta)=\mathcal{L}^{(2)}(\vec{x}, \eta)+\frac{d \mathcal{D}}{d \eta}, \quad \mathcal{D}=\frac{\mathcal{H}}{2} \psi^{2} .
$$

The wave-functional computed with (14) only differs from the one computed with (13) by a field-dependent phase, namely

$$
\Phi^{(3)}[\psi]=e^{i \mathcal{D}(\psi, \eta)} \Phi^{(2)}[\psi] .
$$


The expectation value of a generic observable $\mathcal{O}[\hat{\psi}, \hat{\pi}]$ can be computed using either $\Phi^{(2)}[\psi]$ or $\Phi^{(3)}[\psi]$. However, if we compute such an expectation value using $\Phi^{(3)}[\psi]$, we have to bear in mind that the canonical momentum $(\hat{\pi})$ acts non-trivially on it, its action being fully specified by the transformation (11). The conclusion is that the expectation value of any operator $\mathcal{O}[\hat{\psi}, \hat{\pi}]$ is independent on the Hamiltonian one is using (we shall see below an example of this fact in the context of cosmological perturbations). In spite of the above equivalence, the state minimizing one of the Hamiltonians at the initial time $\eta_{0}$ does depend on which one of (6)-(8) is chosen. Before discussing the effect of choosing different initial states, let us discuss the Heisenberg-picture evolution of the operators that holds for any choice of the Hamiltonian.

Working in Fourier space,

$$
\hat{\psi}(\vec{x}, \eta)=\frac{1}{(2 \pi)^{3 / 2}} \int d^{3} k \hat{\psi}_{\vec{k}}(\eta) e^{-i \vec{k} \cdot \vec{x}}, \quad \hat{\pi}(\vec{x}, \eta)=\frac{1}{(2 \pi)^{3 / 2}} \int d^{3} k \hat{\pi}_{\vec{k}}(\eta) e^{-i \vec{k} \cdot \vec{x}},
$$

with $\hat{\psi}_{\vec{k}}=\hat{\psi}_{-\vec{k}}^{\dagger}, \hat{\pi}_{\vec{k}}=\hat{\pi}_{-\vec{k}}^{\dagger}$, the evolution in the Heisenberg picture reads

$$
\left(\begin{array}{c}
\hat{\psi}_{\vec{k}}(\eta) \\
\hat{\pi}_{\vec{k}}(\eta)
\end{array}\right)=\left(\begin{array}{cc}
A_{\vec{k}}\left(\eta, \eta_{0}\right) & B_{\vec{k}}\left(\eta, \eta_{0}\right) \\
C_{\vec{k}}\left(\eta, \eta_{0}\right) & D_{\vec{k}}\left(\eta, \eta_{0}\right)
\end{array}\right)\left(\begin{array}{c}
\hat{\psi}_{\vec{k}}\left(\eta_{0}\right) \\
\hat{\pi}_{\vec{k}}\left(\eta_{0}\right)
\end{array}\right),
$$

where

$$
\begin{aligned}
& A_{\vec{k}}\left(\eta, \eta_{0}\right)=i\left[g_{k}\left(\eta_{0}\right) f_{k}^{\star}(\eta)-g_{k}^{\star}\left(\eta_{0}\right) f_{k}(\eta)\right], B_{\vec{k}}\left(\eta, \eta_{0}\right)=i\left[f_{k}(\eta) f_{k}^{\star}\left(\eta_{0}\right)-f_{k}^{\star}(\eta) f_{k}\left(\eta_{0}\right)\right], \\
& C_{\vec{k}}\left(\eta, \eta_{0}\right)=i\left[g_{k}\left(\eta_{0}\right) g_{k}^{\star}(\eta)-g_{k}^{\star}\left(\eta_{0}\right) g_{k}(\eta)\right], D_{\vec{k}}\left(\eta, \eta_{0}\right)=i\left[g_{k}(\eta) f_{k}^{\star}\left(\eta_{0}\right)-g_{k}^{\star}(\eta) f_{k}\left(\eta_{0}\right)\right](18)
\end{aligned}
$$

In Eqs. (18) $f_{k}(\eta)$ and $g_{k}(\eta)$ denote the mode functions that are, respectively, solutions of the Heisenberg evolution equations for a (generic) pair $(\hat{\psi}, \hat{\pi})$ of canonically conjugated operators. At every time, for consistency with the canonical commutation relations, the phases and amplitudes of the mode functions are subjected to the Wronskian condition

$$
f_{k}(\eta) g_{k}^{\star}(\eta)-f_{k}^{\star}(\eta) g_{k}(\eta)=i .
$$

In Eqs. (18), with the condition (19), for $\eta \rightarrow \eta_{0}, C_{\vec{k}}\left(\eta_{0}, \eta_{0}\right)=B_{\vec{k}}\left(\eta_{0}, \eta_{0}\right)=0$ and $A_{\vec{k}}\left(\eta_{0}, \eta_{0}\right)=D_{\vec{k}}\left(\eta_{0}, \eta_{0}\right)=1$. Clearly, each of the Hamiltonians (6)-(8) leads to different $f_{k}(\eta)$ and $g_{k}(\eta)$ all satisfying (19).

The time $\eta_{0}(k)$ will be on the NPH (see below) and different Hamiltonians will be minimized at that time. It should already be clear at this point that the two-point function of, say, $\hat{\psi}_{\vec{k}}$ will depend on the choice of the initial state through Eqs. (17) and (18), since minimizing different Hamiltonians corresponds to imposing different conditions on the field operators at $\eta_{0}(k)$.

In order to deal with simple mode functions, we will confine our attention to the case of an inflationary background with a scale factor parametrized, in conformal time, as

$$
a(\eta)=\left(-\frac{\eta}{\eta_{1}}\right)^{-\beta}, \quad \eta<-\eta_{1}
$$


where $\eta_{1}$ marks the end of the inflationary epoch. The pure de Sitter case corresponds to $\beta=1$. The inequality $k / a(\eta) \leq \Lambda$ is saturated, by definition, at the time $\eta_{0}(k)$. We shall refer to this time as the time of exit from the NPH, not to be confused, of course, with the more standard "horizon-exit" time $\eta_{\text {ex }}$. In our case,

$$
\eta_{0}(k)=-\eta_{1}\left(\frac{\Lambda}{k}\right)^{1 / \beta}
$$

Let us then discuss the minimization of the different Hamiltonians starting with (6) but suppressing the label ${ }^{(1)}$ for simplicity. In Fourier space

$$
\hat{H}_{k}(\eta)=\frac{1}{4}\left[\frac{1}{a^{2}}\left(\hat{\Pi}_{\vec{k}} \hat{\Pi}_{\vec{k}}^{\dagger}+\hat{\Pi}_{\vec{k}}^{\dagger} \hat{\Pi}_{\vec{k}}\right)+k^{2} a^{2}\left(\hat{\Psi}_{\vec{k}} \hat{\Psi}_{\vec{k}}^{\dagger}+\hat{\Psi}_{\vec{k}}^{\dagger} \hat{\Psi}_{\vec{k}}\right)\right]
$$

with $\hat{H}(\eta)=\int d^{3} k \hat{H}_{k}(\eta)$. The appropriately normalized mode functions are

$$
\begin{aligned}
f_{k}(\eta) & =\frac{\mathcal{N}}{a(\eta) \sqrt{2 k}} \sqrt{-x} H_{\mu}^{(1)}(-x), \quad g_{k}(\eta)=a^{2}(\eta) f_{k}^{\prime}, \\
\mathcal{N} & =\sqrt{\frac{\pi}{2}} e^{\frac{i}{2}(\mu+1 / 2) \pi}, \quad \beta=\mu-\frac{1}{2},
\end{aligned}
$$

where $x=k \eta$, and $H_{\mu}^{(1)}(-x)$ are the first-order Hankel functions of index $\mu$ [17]. In order to minimize the Hamiltonian (6) let us consider the auxiliary operator

$$
\hat{Q}_{\vec{k}}=\frac{1}{\sqrt{2 k}}\left[\frac{\hat{\Pi}_{\vec{k}}}{a}-i a k \hat{\Psi}_{\vec{k}}\right] .
$$

Equation (25) allows Eq. (22) to be expressed as

$$
\hat{H}_{k}=\frac{k}{2}\left[\hat{Q}_{\vec{k}}^{\dagger} \hat{Q}_{\vec{k}}+\hat{Q}_{\vec{k}} \hat{Q}_{\vec{k}}^{\dagger}\right],
$$

while canonical commutation relations between conjugate field operators,

$$
[\hat{\psi}(\vec{x}, \eta), \hat{\pi}(\vec{y}, \eta)]=i \delta^{(3)}(\vec{x}-\vec{y}),
$$

imply $\left[\hat{Q}_{\vec{k}}, \hat{Q}_{\vec{p}}^{\dagger}\right]=\delta^{(3)}(\vec{k}-\vec{p})$. Consequently, the state minimizing (6) is the one annihilated by $\hat{Q}_{\vec{k}}$ (provided it is normalizable).

In order to evaluate the corrections induced on the two-point function by this particular initial state, we have to compute

$$
\left\langle 0^{(1)}, \eta_{0}|\hat{h}(\vec{x}, \eta) \hat{h}(\vec{y}, \eta)| \eta_{0}, 0^{(1)}\right\rangle \equiv \frac{\ell_{\mathrm{P}}^{2}}{4 \pi^{3}} \int d^{3} k \int d^{3} p\left\langle\hat{\Psi}_{\vec{k}}(\eta) \hat{\Psi}_{\vec{p}}(\eta)\right\rangle e^{-i(\vec{k} \cdot \vec{x}+\vec{p} \cdot \vec{y})},
$$

where $\langle\ldots\rangle \equiv\left\langle 0^{(1)}, \eta_{0}|\ldots| \eta_{0}, 0^{(1)}\right\rangle$ means that the expectation values should be evaluated over the state minimizing $H^{(1)}$ at the time $\eta_{0}$. Inserting Eqs. (17) into Eq. (28), we obtain

$$
\begin{aligned}
& \left\langle 0^{(1)}, \eta_{0}|\hat{h}(\vec{x}, \eta) \hat{h}(\vec{y}, \eta)| \eta_{0}, 0^{(1)}\right\rangle=\frac{\ell_{\mathrm{P}}^{2}}{4 \pi^{3}} \int d^{3} k \int d^{3} p\left[A_{k}\left(\eta, \eta_{0}\right) A_{p}\left(\eta, \eta_{0}\right)\left\langle\hat{\Psi}_{\vec{k}}\left(\eta_{0}\right) \hat{\Psi}_{\vec{p}}\left(\eta_{0}\right)\right\rangle\right. \\
& +B_{k}\left(\eta, \eta_{0}\right) B_{p}\left(\eta, \eta_{0}\right)\left\langle\hat{\Pi}_{\vec{k}}\left(\eta_{0}\right) \hat{\Pi}_{\vec{p}}\left(\eta_{0}\right)\right\rangle+B_{k}\left(\eta, \eta_{0}\right) A_{p}\left(\eta, \eta_{0}\right)\left\langle\hat{\Pi}_{\vec{k}}\left(\eta_{0}\right) \hat{\Psi}_{\vec{p}}\left(\eta_{0}\right)\right\rangle+ \\
& \left.+A_{k}\left(\eta, \eta_{0}\right) B_{p}\left(\eta, \eta_{0}\right)\left\langle\hat{\Psi}_{\vec{k}}\left(\eta_{0}\right) \hat{\Pi}_{\vec{p}}\left(\eta_{0}\right)\right\rangle\right] e^{-i(\vec{k} \cdot \vec{x}+\vec{p} \cdot \vec{y})}
\end{aligned}
$$


The various expectation values appearing in (29) can be computed using the relation between the canonical operators (evaluated at the initial time $\eta_{0}$ ) and the operators $(25)$ annihilating the initial state. Defining now the power spectrum (i.e. the Fourier transform of the two-point function) by,

$$
\left\langle 0^{(1)}, \eta_{0}|\hat{h}(\vec{x}, \eta) \hat{h}(\vec{y}, \eta)| \eta_{0}, 0^{(1)}\right\rangle=\int \frac{d k}{k}\left|\delta_{h}(k, \eta)\right|^{2} \frac{\sin k r}{k r},
$$

we obtain, from Eq. (29) and with the help of Eqs. (23)-(24),

$$
\left|\delta_{h}(k, \eta)\right|^{2}=\frac{2^{4 \beta-1}}{\pi^{3}}(2 \beta)^{-2 \beta} \Gamma(\beta+1 / 2)^{2}\left(\frac{H_{1}}{M_{\mathrm{P}}}\right)^{2}\left(\frac{k}{k_{1}}\right)^{2(1-\beta)}\left[1+\frac{\beta}{x_{0}} \sin \left(2 x_{0}+\beta \pi\right)\right],
$$

where we denote by $H_{1}$ the Hubble parameter at the end of inflation. Finally, $\frac{k}{k_{1}}=\frac{\omega}{\omega_{1}}$ is the ratio of the generic proper frequency to the one corresponding to the end-point of the spectrum $\omega_{1}=H_{1} / a$.

In order to derive Eq. (31) the limit $x=k \eta \ll 1$, corresponding to looking at the correlation function at late times, has been taken. Also, since $\left|x_{0}\right| \gg 1$, only the leading correction in $1 / x_{0}$ has been kept. Furthermore, using $k / a\left(\eta_{0}\right)=\Lambda$, according to Eq. (20) the initial time $\eta_{0}$ can be easily related to the value of $\Lambda$ by

$$
\left|x_{0}\right|=\left|k \eta_{0}\right|=\beta \frac{\Lambda}{H_{\mathrm{ex}}^{\mathrm{NPH}}},
$$

where $H_{\mathrm{ex}}^{\mathrm{NPH}}=H\left(t_{0}(k)\right)$ denotes the Hubble parameter at the time $t_{0}(k)$ when a given scale "exits" the NPH. Note that $x_{0}$ depends on $k$ except in the case of pure de Sitter. We see that, as a consequence, corrections to the standard results are larger at small $k$ for power law inflation (corresponding to larger values of $H_{\mathrm{ex}}^{\mathrm{NPH}}$ ), while the opposite is true for superinflation $(0<\beta<1)$. Equation $(31)$ generalizes the result obtained in $[7]$ in the case of pure de Sitter space. Indeed, for $\beta=1$, Eq. (31) gives exactly

$$
\left|\delta_{h}(k, \eta)\right|^{2}=\frac{1}{2 \pi^{2}}\left(\frac{H}{M_{\mathrm{P}}}\right)^{2}\left[1-\frac{\sin 2 x_{0}}{x_{0}}\right] .
$$

Let us now repeat the same procedure in the case of $H^{(2)}$, which is, incidentally, the Hamiltonian used in [7]. In the case of (7) we have

$$
\hat{H}_{k}(\eta)=\frac{1}{4}\left[\left(\hat{\pi}_{\vec{k}} \hat{\pi}_{\vec{k}}^{\dagger}+\hat{\pi}_{\vec{k}}^{\dagger} \hat{\pi}_{\vec{k}}\right)+k^{2}\left(\hat{\psi}_{\vec{k}} \hat{\psi}_{\vec{k}}^{\dagger}+\hat{\psi}_{\vec{k}}^{\dagger} \hat{\psi}_{\vec{k}}\right)+k F(x)\left(\hat{\pi}_{\vec{k}} \hat{\psi}_{\vec{k}}^{\dagger}+\hat{\pi}_{\vec{k}}^{\dagger} \hat{\psi}_{\vec{k}}+\hat{\psi}_{\vec{k}} \hat{\pi}_{\vec{k}}^{\dagger}+\hat{\psi}_{\vec{k}}^{\dagger} \hat{\pi}_{\vec{k}}\right)\right],
$$

where

$$
k F(x)=\mathcal{H} .
$$

Solving the evolution in the Heisenberg picture, the mode functions can be written as [17]

$$
\begin{aligned}
f_{k}(\eta) & =\frac{\mathcal{N}}{\sqrt{2 k}} \sqrt{-x} H_{\mu}^{(1)}(-x), \\
g_{k}(\eta) & =-\mathcal{N} \sqrt{\frac{k}{2}} \sqrt{-x} H_{\mu-1}^{(1)}(-x) .
\end{aligned}
$$


In order to minimize the Hamiltonian (34) at the initial time $\eta_{0}$, we introduce

$$
\hat{Q}_{\vec{k}}=\frac{1}{\sqrt{2 k}}\left[e^{-i \gamma} \hat{\pi}_{\vec{k}}-i e^{i \gamma} k \hat{\psi}_{\vec{k}}\right]
$$

where $\gamma$ is a time-dependent parameter. Using Eq. (38), the Hamiltonian (34) can be put in the same form as (26) provided the following relation is imposed between $\gamma$ and $F(x)$ of Eq. (34):

$$
\sin 2 \gamma=F(x) .
$$

The canonical commutation relations Eq. (27) now imply $\left[\hat{Q}_{\vec{k}}, \hat{Q}_{\vec{p}}^{\dagger}\right]=\cos 2 \gamma \delta^{(3)}(\vec{k}-\vec{p})$, so that the initial state minimizing (34) is again the one annihilated by $\hat{Q}_{\vec{k}}$.

The wave-functional of the initial state can be easily derived and, for each mode, it has a Gaussian form:

$$
\Phi\left[\psi_{\vec{k}}\right]=N \exp \left(-\sum_{k} \frac{k}{2}\left(\psi_{\vec{k}} \psi_{-\vec{k}}\right) e^{-2 i \gamma}\right) .
$$

This state is normalizable provided $|\gamma|<\pi / 4$. Using Eq. (39), we see that $|\gamma|=\pi / 4$ corresponds to a time $\eta_{0}$ for which $\left|F\left(x_{0}\right)\right|=1$, which is basically equivalent to the condition of (standard) horizon crossing. Consequently, provided the modes of the field are inside the horizon at the "initial" time $\eta_{0}$, the state (40) is normalizable.

The two-point function to be computed now is

$$
\left\langle 0^{(2)}, \eta_{0}|\hat{h}(\vec{x}, \eta) \hat{h}(\vec{y}, \eta)| \eta_{0}, 0^{(2)}\right\rangle=\frac{\ell_{\mathrm{P}}^{2}}{4 \pi^{3} a(\eta)^{2}} \int d^{3} k \int d^{3} p\left\langle\hat{\psi}_{\vec{k}}(\eta) \hat{\psi}_{\vec{p}}(\eta)\right\rangle e^{-i(\vec{k} \cdot \vec{x}+\vec{p} \cdot \vec{y})}
$$

and the related power spectrum evaluated at late times $(x=k \eta \ll 1)$ is

$$
\left|\delta_{h}(k, \eta)\right|^{2}=\frac{2^{4 \beta-1}}{\pi^{3}}(2 \beta)^{-2 \beta} \Gamma(\beta+1 / 2)^{2}\left(\frac{H_{1}}{M_{\mathrm{P}}}\right)^{2}\left(\frac{k}{k_{1}}\right)^{2(1-\beta)}\left[1-\beta \frac{\cos \left(2 x_{0}+\beta \pi\right)}{2 x_{0}^{2}}\right] .
$$

In the de Sitter case, $\beta=1$, Eq. (42) gives

$$
\left|\delta_{h}(k, \eta)\right|^{2}=\frac{1}{2 \pi^{2}}\left(\frac{H}{M_{P}}\right)^{2}\left[1+\frac{\cos 2 x_{0}}{2 x_{0}^{2}}\right] .
$$

Note that, in [7], use was made of the Hamiltonian $H^{(2)}$, but the initial state was such as to minimize $H^{(1)}$. Thus, in agreement with our general statements, the results found in [7], were the same (in de Sitter) as those we found unsing $H^{(1)}$ and minimizing it. What matters is not which Hamiltonian is used for the evolution, but which one is used for defining the initial state through an energy minimization procedure!

The Hamiltonian (8) can be minimized following the same procedure already discussed in the case of Eqs. (6) and (7). Defining the function

$$
\omega^{2}(x)=\left(1-\frac{1}{k^{2}} \frac{a^{\prime \prime}}{a}\right)
$$


the Hamiltonian (8) can be written in the simple form ${ }^{3}$

$$
\hat{H}_{k}(\eta)=\frac{1}{4}\left[\left(\hat{\pi}_{\vec{k}} \hat{\pi}_{\vec{k}}^{\dagger}+\hat{\pi}_{\vec{k}}^{\dagger} \hat{\pi}_{\vec{k}}\right)+k^{2} \omega^{2}(x)\left(\hat{\psi}_{\vec{k}} \hat{\psi}_{\vec{k}}^{\dagger}+\hat{\psi}_{\vec{k}}^{\dagger} \hat{\psi}_{\vec{k}}\right)\right] .
$$

Defining now the operator

$$
\hat{Q}_{\vec{k}}=\frac{1}{\sqrt{2 k}}\left[\hat{\pi}_{\vec{k}}-i k \omega \hat{\psi}_{\vec{k}}\right]
$$

the Hamiltonian can again be expressed in the same form previously discussed, namely, the one given by Eq. (26) with the caveat that now the operator (46), if compared to the one defined in Eq. (38) has a different expression in terms of the canonical fields. The commutation relations are now $\left[Q_{\vec{k}}, Q_{\vec{p}}^{\dagger}\right]=\omega \delta^{(3)}(\vec{k}-\vec{p})$. The mode functions $f_{k}(\eta)$ are the same as the ones given in Eq.(36), while $g_{k}$ is given by

$$
g_{k}(\eta)=-\mathcal{N} \sqrt{\frac{k}{2}} \sqrt{-x}\left[H_{\mu-1}^{(1)}(-x)+\frac{(1-2 \mu)}{2(-x)} H_{\mu}^{(1)}(-x)\right]
$$

Repeating the steps used in the two previous cases we arrive at the power spectrum:

$$
\left|\delta_{h}(k, \eta)\right|^{2}=\frac{2^{4 \beta-1}}{\pi^{3}}(2 \beta)^{-2 \beta} \Gamma(\beta+1 / 2)^{2}\left(\frac{H_{1}}{M_{\mathrm{P}}}\right)^{2}\left(\frac{k}{k_{1}}\right)^{2(1-\beta)}\left[1-\frac{\beta(\beta+1)}{2 x_{0}^{3}} \sin \left(2 x_{0}+\pi \beta\right)\right] .
$$

In the de Sitter case, we have:

$$
\left|\delta_{h}(k, \eta)\right|^{2}=\frac{1}{2 \pi^{2}}\left(\frac{H}{M_{\mathrm{P}}}\right)^{2}\left[1+\frac{\sin 2 x_{0}}{x_{0}^{3}}\right] .
$$

Comparing Eq. (48) with (42) we see that the corrections are even smaller than the ones obtained using the Hamiltonian of Eq. (34). Furthermore, both Eqs. (48) and (42) lead to effects smaller than (31).

We have shown that transplanckian effects can be tamed by choosing a sufficiently adiabatic Hamiltonian. We may ask whether the opposite can be achieved, i.e. enhance transplanckian effects by minimizing particularly contrived Hamiltonians on the NPH. The answer turns out to be positive. Consider the following generating functional

$$
\mathcal{F}_{3 \rightarrow 4}(\psi, \tilde{\Pi})=\int d^{3} x\left[\psi \tilde{\Pi}-\frac{\tilde{\Pi}^{2}}{2 \mathcal{H}}\right] .
$$

This rather weird canonical transformation allows the passage from $H^{(3)}$ to an $H^{(4)}$, where the new fields $\tilde{\Psi}$ and the new momenta $\tilde{\Pi}$ are

$$
\tilde{\Psi}=\psi-\frac{\tilde{\Pi}}{\mathcal{H}}, \quad \tilde{\Pi}=\tilde{\pi} .
$$

\footnotetext{
${ }^{3}$ From now on the tilde in the momentum operators will be omitted for the sake of simplicity.
} 
Without going through the details of the derivation, the following highly "transplanckian" Hamiltonian can be obtained:

$$
H^{(4)}=\int d^{3} x \frac{1}{2}\left[\frac{k^{2}}{\mathcal{H}^{2}} \tilde{\Pi}^{2}+2\left(\frac{k^{2}}{\mathcal{H}}-\mathcal{H}-\frac{\mathcal{H}^{\prime}}{\mathcal{H}}\right) \tilde{\Pi} \tilde{\Psi}+\left(k^{2}-\frac{a^{\prime \prime}}{a}\right) \tilde{\Psi}^{2}\right] .
$$

The same procedure as described for the other three Hamiltonians can be repeated, and the correction induced in the power spectrum turns out to be of order 1 in $1 / x_{0}$, namely (here we just give the result in the de Sitter case):

$$
\left|\delta_{h}(k, \eta)\right|^{2}=\frac{1}{4 \sqrt{2} \pi^{2}}\left(\frac{H}{M_{\mathrm{P}}}\right)^{2}\left[3-\cos 2 x_{0}\right] .
$$

The question thus arises of which Hamiltonian, if any, should be minimized on the NPH. A similar problem has been discussed in the past [18, 19], though in a somewhat different context. The idea was to study the properties of two-point functions at arbitrary space-time points in the state $\left|0, t_{0}\right\rangle$, minimizing a certain Hamiltonian at a given (mode-independent) "initial time" time $t_{0}$, i.e.

$$
\left\langle t_{0}, 0|\hat{h}(x) \hat{h}(y)| 0, t_{0}\right\rangle,
$$

where $x \equiv(\vec{x}, \eta)$ and $y \equiv\left(\vec{y}, \eta^{\prime}\right)$. It was found [18] that, only if the state $\left|0, t_{0}\right\rangle$ minimizes a very special class of Hamiltonians (in our case just $H^{(3)}$ ), do the singularities in (54) lie on the light cone $(x-y)^{2}=0$ and are of the Hadamard form. All other Hamiltonians lead to additional singularities outside the light cone.

It is easy to generalize our discussion to two-point functions at unequal times. However, the situation discussed in [18] is quite different from the one described here. On the one hand we do impose a momentum cut-off. By itself, this would only smooth out the singularity without really removing the phenomenon. A more significant distinction is that we minimize different pieces of the Hamiltonian at different times. Direct calculations show that, unfortunately, Hamiltonians (7) and (8) cannot be distinguished by using the singularity arguments of [18].

There is probably a more physical reason why (45) should be preferred over (34). In the case of (34) the "interacting" part of the Hamiltonian vanishes as $1 / \eta$ for large $\eta$. In other words, the switching-on of the interaction at finite $\eta$ is faster for $H^{(2)}$ than it is for $H^{(3)}$. The latter is definitely a more "adiabatic" Hamiltonian. It thus looks reasonable to assume that the truly initial state remains in the ground state of this "very adiabatic" Hamiltonian.

Our conclusions can be summarized as follows:

- In case where one has serious doubts about the theory of cosmological perturbations at $t \rightarrow-\infty$ it looks reasonable to assign "initial" quantum fluctuation on a NPH to the future of which the standard procedure applies.

- Although there are ambiguities in defining the Hamiltonian in time-dependent problems, physical results do not depend on the choice of such Hamiltonian. Corrections to the standard result only depend on the choice of the "initial" state on the NPH. 
- An attractive, though by no means unique, choice for the initial state is the one that minimized the Hamiltonian on the NPH. The so-defined state does depend on how the Hamiltonian to be minimized is chosen.

- The size of transplanckian corrections is a sensitive function of that choice with the most "adiabatic" Hamiltonians giving the smallest corrections in terms of the small parameter $H_{\mathrm{ex}}^{\mathrm{NPH}} / \Lambda$. The most adiabatic Hamiltonian, whose minimization on a space-like hypersurface leads to correlation functions of the Hadamard form, gives transplanckian corrections that are much smaller than previously suggested.

- A final answer to the question of transplanckian corrections will only be found when a theory of transplanckian physics is defined, ... and solved.

It is a pleasure to thank M. Gasperini for useful discussions and correspondence. V.B. would like to thank the Theory Division of CERN for hospitality while this work was carried out.

\section{References}

[1] H. Kodama and M. Sasaki, Int. J. Mod. Phys. A1, 265 (1986).

[2] A. Kempf and J. C. Niemeyer Phys.Rev.D 64, 103501 (2001).

[3] R. Easther, B. Greene, W. Kinney and G. Shiu, Phys. Rev.D 64, 103502 (2001).

[4] R. Brandenberger and J. Martin Phys. Rev.D 65, 103514 (2002).

[5] N. Kaloper, M. Kleban, A. Lawrence, and S. Shenker Phys. Rev.D 66, 123510 (2002).

[6] A. Starobinsky and I. Tkachev, JETP Lett.76 2352002 [Pisma Zh. Eksp. Teor. Fiz.76, $2912002]$.

[7] U. Danielsson, Phys.Rev.D 66, 023511 (2002)

[8] G. Veneziano, String Inspired Alternatives to Standard Inflation, invited talk at the XXI Texas Symposium, Florence, Italy (Dec. 2002), to appear.

[9] M. Gasperini and G. Veneziano, Phys. Rept. 373, 1 (2003).

[10] L. Bergstrom and U. Danielsson, JHEP 0212, 038 (2002).

[11] C. Burgess, J. Cline, F. Lemieux and R. Holman, hep-th/0210233.

[12] L. Mersini, M. Bastero-Gil, P. Kanti, Phys.Rev.D 64, 043508 (2001).

[13] K. Goldstein and D. Lowe, hep-th/0208167. 
[14] N. Kaloper, M. Kleban, A. Lawrence, S. Shenker and L. Susskind, JHEP 0211, 037 (2002).

[15] L. P. Grishchuk, Sov. Phys. JETP 40409 (1975); L. P. Grishchuk and M. Solokhin, Phys. Rev. D 432566 (1991).

[16] A. L. Matacz, Phys. Rev. D 49, 788 (1994).

[17] A. Erdelyi, W. Magnus, F. Obehettinger, and F. R. Tricomi, Higher Trascendental Functions (McGraw-Hill, New York, 1953).

[18] N. Weiss, Phys. Rev. D 34, 1768 (1986).

[19] S. Fulling, Gen. Relativ. Gravit. 10, 807 (1979).

[20] V.F. Mukhanov, H.A. Feldman, and R. H. Brandenberger, Phys. Rep. 215, 203 (1992). 